
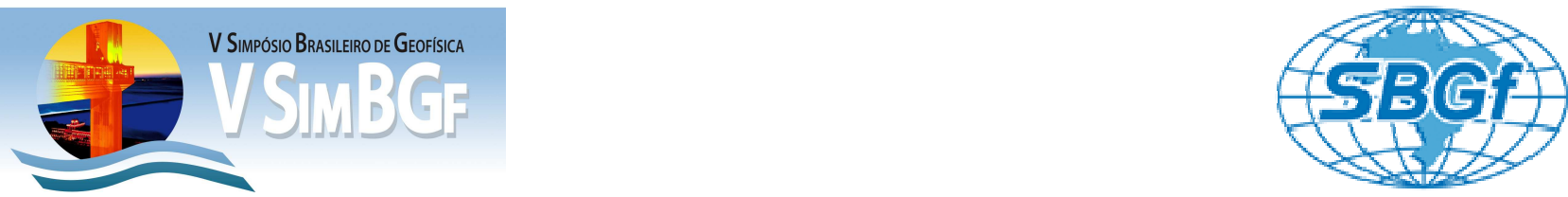

\title{
Campo termal da litosfera da Região Norte de Minas Gerais
}

Évilla Azevedo Schirmer* ${ }^{* 1}$ - evillazevedo@hotmail.com

Carlos Henrique Alexandrino', 'Universidade Federal dos Vales do Jequitinhonha e Mucuri - UFVJM - Campus do Mucuri Teófilo Otoni - MG

Copyright 2012, SBGf - Sociedade Brasileira de Geofísica

Este texto foi preparado para a apresentação no V Simpósio Brasileiro de Geofísica, Salvador, 27 a 29 de novembro de 2012. Seu conteúdo foi revisado pelo Comitế Técnico do V SimBGf, mas não necessariamente representa a opinião da SBGf ou de seus associados. É proibida a reprodução total ou parcial deste material para propósitos comerciais sem prévia autorização da SBGt.

\section{Resumo}

O presente trabalho teve como enfoque a caracterização do campo termal da litosfera da Região Norte do estado de Minas Gerais. Utilizando dados existentes na literatura para a condutividade térmica, o calor radiogênico, o fator de decaimento exponencial e a temperatura na superfície, realizaram-se a estimativa dos valores de temperatura em função da profundidade, gradiente e fluxos geotérmicos para a região estudada. Os valores encontrados foram satisfatórios e os mapas dos resultados permitiram visualizar a relação entre as províncias geológicas e o campo termal na litosfera terrestre, como por exemplo, altos valores de Fluxo Térmico para a área do Complexo Porteirinha, que é uma província diamantífera da região.

\section{Introdução}

A evolução das formas atuais de elevação dos continentes, bem como a ocorrência das atividades sísmicas são conseqüências diretas dos processos térmicos interiores da Terra. Desta forma, o conhecimento do regime térmico é fundamental para o estudo da dinâmica e da evolução do nosso planeta. Entre outras medidas geotérmicas, a determinação do gradiente de temperatura e do fluxo geotérmico é primordial na caracterização das camadas interiores. As propriedades químicas e físicas dos materiais que constituem o nosso planeta são influenciados pelo regime térmico, logo, se faz necessário entender a distribuição do fluxo de calor terrestre.

A maior parte, da região Norte do Estado de Minas Gerais encontra-se localizada na Província Estrutural do São Francisco, mais precisamente dentro Craton de mesmo nome. Situada na região oriental da plataforma Sul-Americana, ela ocupa uma área de aproximadamente $128.460 \mathrm{~km}^{2}$. A figura (1) mostra suas principais formações geológicas.

Dentre as suas principais formações destacam-se: a Formação Urucuia de idade cretácica, constituída por arenitos finos de sedimentação eólica (Chaves et al. 2011).; o Orógeno Araçuaí tem como principal unidade estratigráfica 0 Grupo Macaúbas, de idade neoproteozóica, constituído por quartzitos e metadiamictitos (Martins 2006). Grupo Macaúbas e, em sua maior parte, pela cobertura neoproteozóica do Grupo Bambuí (Iglesias \& Uhlein 2009); o Complexo Porteirinha, caracterizado por associações rochosas predominantemente arqueanas, é majoritariamente constituído por ortognaisses e ocupa uma posição mais externa do Orógeno Araçuaí (Noce 2007); e o Supergrupo Espinhaço compõe e sustenta a serra homônima e se estende por aproximadamente $1200 \mathrm{~km}$, distribuindo-se ao longo do Orógeno Araçuaí ou como cobertura sobre o atual Cráton do São Francisco. (Martins et al. 2008). No sentido estratigráfico, compreende metassedimentos de baixo grau metamórfico formados por meta-sequências siliciclásticas, principalmente arenitos associados a psefitos e pelitos, além de rochas carbonáticas e vulcânicas (Jr. Et al. 2011).

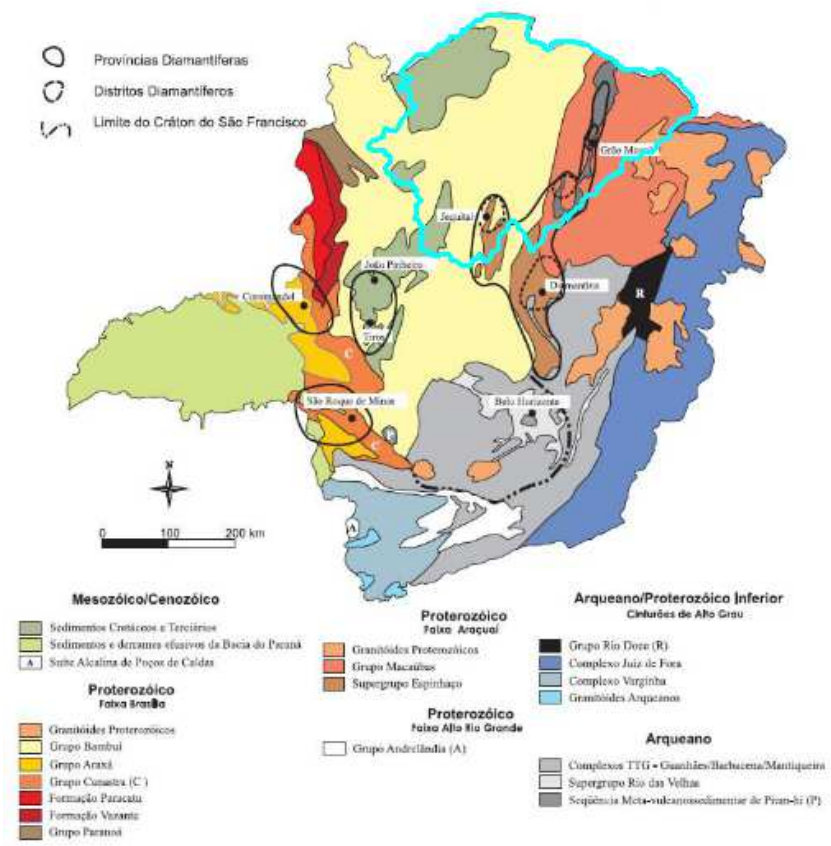

Figura 1 - As províncias geológicas da área de estudo (modificado de Penha et al., 2000).

\section{Metodologia}

$\mathrm{Na}$ análise do campo térmico da litosfera a variação regional do gradiente e do fluxo térmico resultam de um processo de determinação das distribuições verticais de Condutividade Térmica $\lambda$ e produção de Calor Radiogênico $A_{0}$ e da utilização de um modelo que integra os funcionais destes parâmetros e suas características geológicas.

O processo inicial deste trabalho consistiu na localização geográfica e determinação da altitude de cada cidade da Região Norte do estado de Minas Gerais, o que foi feito a partir dos dados disponíveis no site Apollo 11.com (http://www.apolo11.com/). Os dados de Altura Geoidal $(\mathrm{km})$ dos blocos estruturais na área de estudo foram 
extraídos do modelo geopotencial terrestre, conhecido como EGM2008 (Alexandrino \& Hamza 2008). De posse dessas informações, utilizou-se o método da Isostasia Termal, processo geodinâmico em que as variações de elevação são determinadas pelo regime termal da litosfera, para determinar os dados de Espessura Crustal $Z_{c}(\mathrm{~km})$ e Temperatura na Moho $T_{m}\left({ }^{\circ} \mathrm{C}\right)$. As informações sobre a Temperatura na Superfície $T_{\mathrm{ms}}\left({ }^{\circ} \mathrm{C}\right)$ para cada cidade foram obtido no site do Istituto nacional de Meteorologia - INMET.

Baseado nas informações litológicas do Mapa Geológico do estado, da Companhia Mineradora de Minas Gerais COMIG, determinou-se a Condutividade Térmica $\lambda$ $\left(\mathrm{WmK}^{-1}\right)$, tendo como base os resultados das medições experimentais realizadas por Branco da Silva e Hamza (2001), Cerrone e Hamza (2003) e Gomes e Hamza (2005).

Os dados de Calor Radiogênico $A_{0}\left(\mu \mathrm{Wm}^{-3}\right)$ e Fator de Decaimento Exponencial $D(\mathrm{~km})$ utilizados neste trabalho foram retirados da Tese de Doutorado de Alexandrino (2008). Os valores foram calculados a partir da velocidade de ondas sísmicas. As tabelas (1) e (2), apresesta os valores das grandezas geotérmicas obtidas pelo método da isostasia termal.

Tabela 1 - Valores médios da condutividade térmica $(\lambda)$, calor radiogênico, $\left(A_{0}\right)$, e Fator de Decaimento Exponencial D.

\begin{tabular}{|c|c|c|c|}
\hline $\begin{array}{c}\text { Província } \\
\text { Geológica }\end{array}$ & $\begin{array}{c}\mathbf{\lambda} \\
(\mathbf{W} / \mathbf{m ~ K})\end{array}$ & $\begin{array}{c}\mathbf{A}_{\circ} \\
\left(\boldsymbol{\mu} \mathbf{W m}^{-3}\right)\end{array}$ & $\begin{array}{c}\mathbf{D} \\
(\mathbf{k m})\end{array}$ \\
\hline Formação Urucuia & 3.6 & 1.7 & 11.4 \\
\hline Grupo Macaúbas & 2.4 & 1.7 & 11.3 \\
\hline $\begin{array}{c}\text { Complexo } \\
\text { Porteirinha }\end{array}$ & 3.0 & 1.8 & 12.6 \\
\hline Grupo Bambuí & 3.1 & 1.6 & 10.5 \\
\hline $\begin{array}{c}\text { Supergrupo } \\
\text { Espinhaço }\end{array}$ & 2.5 & 1.5 & 12.3 \\
\hline
\end{tabular}

Tabela 2 - Valores médios da espessura crustal ( $\left.\mathrm{Z}_{\mathrm{M}}\right)$, temperatura na superfície $\left(T_{0}\right)$ e temperatura na base da crosta $\left(T_{M}\right)$.

\begin{tabular}{|c|c|c|c|}
\hline $\begin{array}{c}\text { Província } \\
\text { Geológica }\end{array}$ & $\mathbf{z}_{\mathbf{M}}(\mathbf{k m})$ & $\mathbf{T}_{\mathbf{0}}(\mathbf{} \mathbf{C})$ & $\mathbf{T}_{\mathbf{M}}(\mathbf{\circ} \mathbf{C})$ \\
\hline Formação Urucuia & 43.4 & 24.0 & 385 \\
\hline Grupo Macaúbas & 43.4 & 23.8 & 384 \\
\hline $\begin{array}{c}\text { Complexo } \\
\text { Porteirinha }\end{array}$ & 43.8 & 23.8 & 393 \\
\hline Grupo Bambuí & 44.1 & 22.9 & 400 \\
\hline $\begin{array}{c}\text { Supergrupo } \\
\text { Espinhaço }\end{array}$ & 44.1 & 21.3 & 388 \\
\hline
\end{tabular}

Modelo de Temperaturas na Crosta:

No ponto de vista da geotermia a litosfera é definida como a região da Terra onde o modo preponderante de transmissão de calor é a condução, desta forma o ponto de partida para determinação do campo térmico da litosfera é a equação da condução de calor. Considerando geometria unidimensional e regime estacionário, pode-se escrevê-la da seguinte forma:

$$
\frac{d^{2} T}{d z^{2}}=\frac{A_{0}}{\lambda} e^{(-z / D)}
$$

Com as seguintes condições de contorno:

$$
\begin{aligned}
& T(z=0)=T_{0} \\
& T\left(z=z_{m}\right)=T_{m}
\end{aligned}
$$

onde $T$ é a temperatura, $z$ a coordenada na direção vertical. $A_{0}, T_{0}$ e $q_{0}$ são respectivamente produção de calor radiogênico, temperatura e fluxo geotérmico, todos com seus valores observados na superfície, $D$ o parâmetro de decaimento exponencial e $\lambda$ a condutividade térmica.

Integrando duas vezes a equação (1), obtemos:

$$
T(z)=-\frac{A_{0} D^{2}}{\lambda} e^{(-z / D)}+c_{1} z+c_{0}
$$

Aplicando as condições de contorno à equação (3) define-se a função da temperatura em função da profundidade, ou seja, a equação (4):

$$
\begin{aligned}
& T(z)=-\frac{A_{0} D^{2}}{\lambda} e^{(-z / D)}+ \\
& {\left[\left(T_{m}-T_{z}\right)-\frac{A_{0} D^{2}}{\lambda}\left(1-e^{(-z / D)}\right)\right] \frac{z}{z_{m}}+} \\
& T_{0}+\frac{A_{0} D^{2}}{\lambda} \\
& \text { Como são conhecidos os valores das grandezas } \\
& \text { geotérmicas da equação (4) podemos estimar o gradiente } \\
& \text { térmico e o fluxo de calor baseados na Lei de Fourier. }
\end{aligned}
$$

\section{Resultados}

Uma vez calculados os valores da Temperatura, Gradiente e Fluxo Térmicos em função da profundidade crustal, utilizou-se o software gráfico Surfer versão 8 para construir os mapas referentes a estes dados. O método de interpolação empregado foi a Krigagem. 


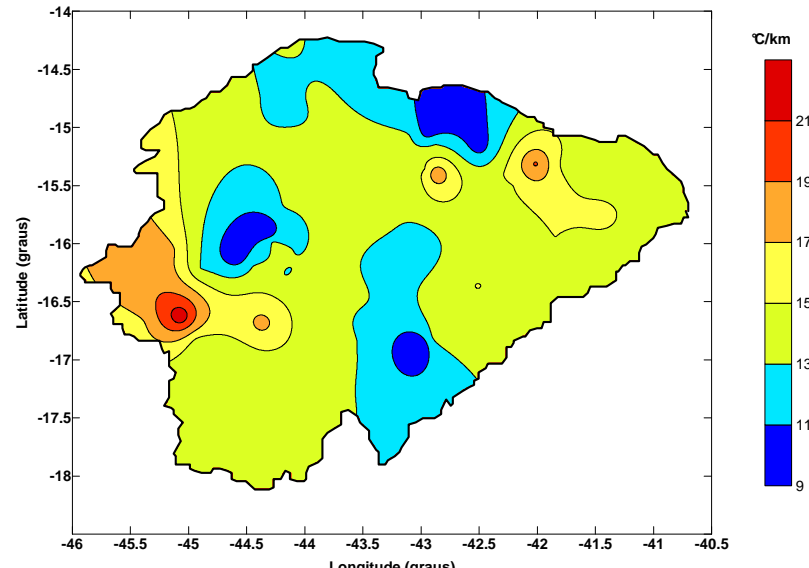

Figura 2 - Gradiente Geotérmico na Superfície da Região Norte.

O mapa da figura (2) apresenta a distribuição do gradiente geotérmico da região Norte. Os maiores valores $\left(>17^{\circ} \mathrm{C} / \mathrm{km}\right)$ encontram-se localizados na parte oeste $e$ os menores valores encontram-se na faixa central. O mapa ainda revela que a maior parte da região possui gradiente em torno de $13^{\circ} \mathrm{C} / \mathrm{km}$.
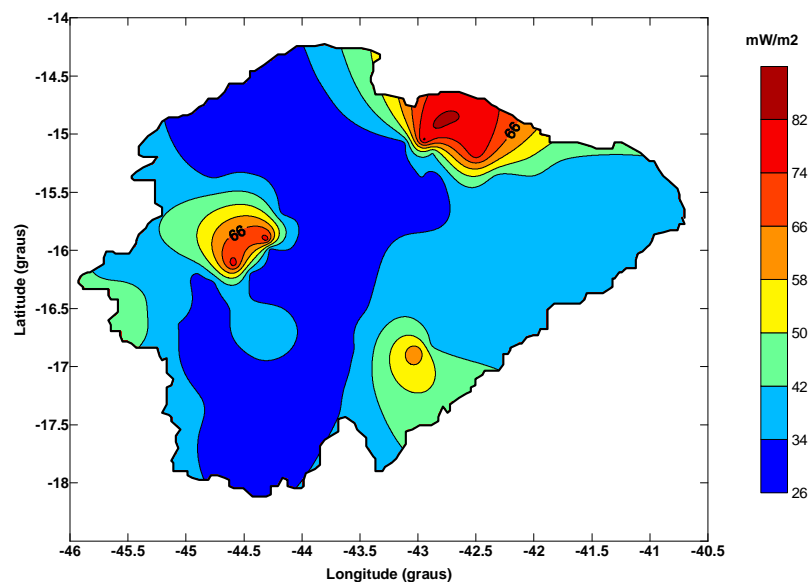

Figura 3 - Fluxo Geotérmico na Superfície da Região Norte.

A figura (3) ilustra a distribuição do Fluxo Geotérmico na Superfície da região Norte. Pela figura é possível observar a diferença no valor do fluxo geotérmico por formação geológica. Os mais baixos valores $(<34$ $\mathrm{mW} / \mathrm{m}^{2}$ ) estão na faixa central. As regiões de maior fluxo $\left(74 \mathrm{~mW} / \mathrm{m}^{2}\right)$.

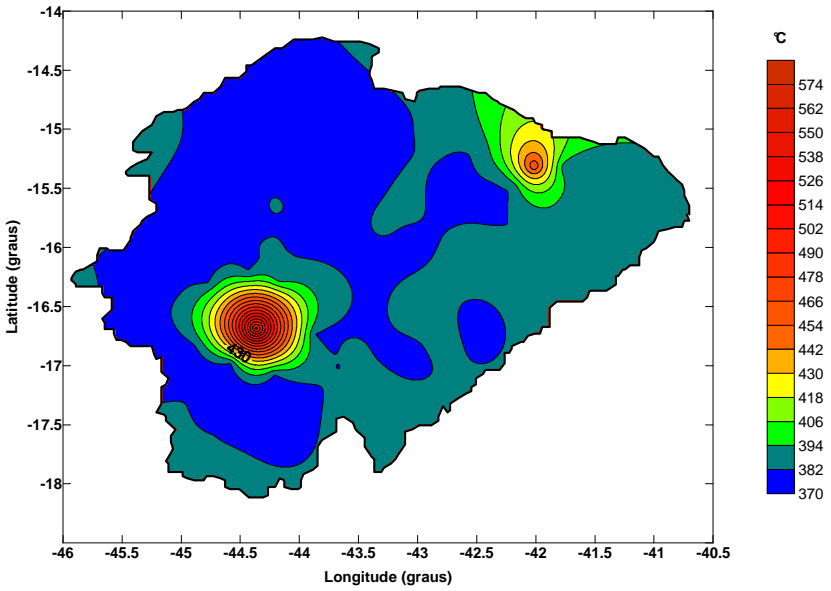

Figura 4 - Temperatura na Moho da Região Norte.

Apresenta-se na figura (4) o mapa de distribuição da Temperatura na Moho para a região Norte. Temperaturas com valores acima de $500{ }^{\circ} \mathrm{C}$, nas regiões nordeste e centro. Nota-se, pela figura 5, que o Fluxo Geotérmico na Moho para a da região. Valores acima de $76 \mathrm{~mW} / \mathrm{m}^{2}$ são encontrados a nordeste e a nordeste. Já os menores, e localiza-se na região oeste e sudeste nodeste podem $\mathrm{Sr}$ encontrados na parte central.

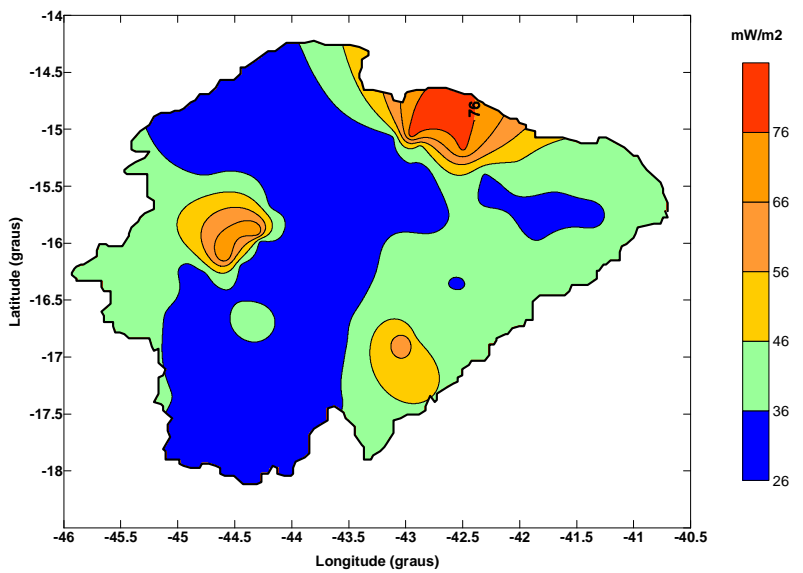

Figura 5 - Fluxo Geotérmico na Moho da Região Norte.

Encontram-se reunidos na tabela (3) os valores médios do gradiente fluxo geotérmico para as principais formações geológicas.

Tabela 3 - Valores médios de gradiente de temperatura (Г) e do fluxo de calor (q) para as principais unidades tectônicas na área de estudo.

\begin{tabular}{|c|c|c|}
\hline $\begin{array}{c}\text { Província } \\
\text { Geológica }\end{array}$ & $\Gamma\left({ }^{0} \mathbf{C} / \mathbf{k m}\right)$ & $\mathbf{q}\left(\mathbf{m W} / \mathbf{m}^{2}\right)$ \\
\hline Formação Urucuia & 13 & 45 \\
\hline Grupo Macaúbas & 15 & 34 \\
\hline $\begin{array}{c}\text { Complexo } \\
\text { Porteirinha }\end{array}$ & 14 & 42 \\
\hline
\end{tabular}




\begin{tabular}{|c|c|c|}
\hline Grupo Bambuí & 13 & 40 \\
\hline $\begin{array}{c}\text { Supergrupo } \\
\text { Espinhaço }\end{array}$ & 14 & 34 \\
\hline
\end{tabular}

\section{Discussão e Conclusões}

As feições representadas pelos mapas estão diretamente relacionadas com a densidade e a distribuição geográfica dos dados, portanto, é possível visualizar as características do regime térmico relacionado com as estruturas geológicas da região de estudo.

Os valores do gradiente e do fluxo geotérmicos são compatíveis com os esperados para região. Os maiores valores para o gradiente geotérmico encontram-se localizados na parte oeste de idade cretácica e na parte nordeste abrangida pelo Complexo Porteirinha de origem arqueana. Os menores valores encontram-se na faixa central e abrange regiões do Grupo Bambuí, e do Grupo Macaúbas.

Os menores valores do fluxo geotérmico estão na faixa central onde se localiza a cobertura neoproteozóica do Grupo Bambuí. A porção leste, compreendida pelo Orógeno Araçuaí e constituída por quartzitos e metadiamictitos é caracterizada por um fluxo entre 34 e $42 \mathrm{~mW} / \mathrm{m}^{2}$. As regiões de maior fluxo correspondem às regiões de formação Urucuia a oeste, Supergrupo Espinhaço a sudeste e Grupo Macaúbas e Complexo Porteirinha a nordeste.

\section{Agradecimentos}

A colaboração da COMIG na aquisição de dados geotérmicos e ao MEC - Ministério de Educação e Cultura pela concessão de bolsa de iniciação científica do PET - Programa de Educação Tutorial para um dos autores.

\section{Referências}

Alexandrino, C.H., 2008. Campo termal da província estrutural São Francisco e faixas móveis adjacentes. Tese de Doutorado em Geofísica, Observatório Nacional, Rio de Janeiro, 2008, 184p.
Alexandrino, C. H., Hamza, V.M., 2008. Avaliação da Isostasia Termal do Estado do Rio de Janeiro e areas de margem Continental Adjacente. Relatório final, Observatório Nacional, Rio de Janeiro, Brasil.

Avilar C., S., 2004. Estrutura Tridimensional da Onda S na Litosfera do Nordeste Brasileiro. Tese de Doutorado, Programa de Pós-Graduação em Geofísica, Observatório Nacional, Rio de Janeiro, Brasil, 273p.

Chaves, M.L.S.C., Andrade, K.W., Benitez, L., 2011. Geologia integrada das Folhas Jequitaí, Bocaiúva e Montes Claros (1:100.000), norte de Minas Gerais. Geonomos, 19(2): 1-7.

Iglesias, M. \& Uhlein, A., 2009. Estratigrafia do Grupo Bambuí e coberturas fanerozóicas no vala do rio São Francisco, norte de Minas. Revista Brasileira de Geociências, 39 (2): 256-266.

Jr., F.C., Dussin, I.A., Martins, M., Santos, M.N., 2011. Nova abordagem tectono-estratigráfica do Supergrupo Espinhaço em sua porção meridional (MG). Geonomos, 19(2): 173-41.

Leite, M.R. \& Brito, J.L.S., 2012. Morphostructural and morfoescultural mapping in the cerrado region in the North of Minas Gerais. Soc. \& Nat., Uberlândia, 24 (1): 115-126.

Martins, M.S., 2006. Geologia dos diamantes e carbonados aluvionares da bacia do rio Macaúbas (MG). Tese de doutoramento, UFMG, Brasil, 269p.

Martins et al. (06 co-authors), 2008. A sequência préglacial do Grupo Macaúbas na área-tipo e o registro da abertura do rifte Araçuaí. Revista Brasileira de Geociências, 38(4): 761-772.

Noce, C.M., Pedrosa-Soares, a.C., Silva, L.C., Alkmim, F.F., 2007. O embasamento arqueano e paleoproterozóico do Orógeno Araçuaí. Geonomos, 15(1): 17-23. 